\title{
High-Resolution Ecological Niche Modeling of Ixodes scapularis Ticks Based on Passive Surveillance Data at the Northern Frontier of Lyme Disease Emergence in North America
}

\author{
Jean-Paul R. Soucy, ${ }^{1-3}$ Andreea M. Slatculescu, Christine Nyiraneza, Nicholas H. Ogden, \\ Patrick A. Leighton, Jeremy T. Kerr, and Manisha A. Kulkarni ${ }^{2}$
}

\begin{abstract}
Background: Lyme disease (LD) is a bacterial infection transmitted by the black-legged tick (Ixodes scapularis) in eastern North America. It is an emerging disease in Canada due to the expanding range of its tick vector. Environmental risk maps for LD, based on the distribution of the black-legged tick, have focused on coarse determinants such as climate. However, climatic factors vary little within individual health units, the level at which local public health decision-making takes place. We hypothesize that high-resolution environmental data and routinely collected passive surveillance data can be used to develop valid models for tick occurrence and provide insight into ecological processes affecting tick presence at fine scales.

Methods: We used a maximum entropy algorithm (MaxEnt) to build a habitat suitability model for I. scapularis in Ottawa, Ontario, Canada using georeferenced occurrence points from passive surveillance data collected between 2013 and 2016 and high-resolution land cover and elevation data. We evaluated our model using an independent tick presence/absence dataset collected through active surveillance at 17 field sites during the summer of 2017.

Results: Our model showed a good ability to discriminate positive sites from negative sites for tick presence $(\mathrm{AUC}=0.878 \pm 0.019$, classification accuracy $=0.835 \pm 0.020)$. Heavily forested suburban and rural areas in the west and southwest of Ottawa had higher predicted suitability than the more agricultural eastern areas.

Conclusions: This study demonstrates the value of passive surveillance data to model local-scale environmental risk for the tick vector of LD at sites of interest to public health. Given the rising incidence of LD and other emerging vector-borne diseases in Canada, our findings support the ongoing collection of these data and collaboration with researchers to provide a timely and accurate portrait of evolving public health risk.
\end{abstract}

Keywords: Borrelia burgdorferi, ecological niche model, GIS, Ixodes scapularis, Lyme disease, passive surveillance

\section{Background}

$\mathbf{L}$ YME DISEASE (LD), caused by the spirochete Borrelia burgdorferi sensu stricto (henceforth termed B. burgdorferi) and transmitted by the black-legged tick (Ixodes scapularis) in eastern North America, is the most common vector-borne disease on the continent and is an emerging disease in Canada (Schauber et al. 2005, Ogden et al. 2015a, 2015 b). Since LD became nationally notifiable in 2009 , the number of cases has increased from $144(0.4$ per 100,000 population) to nearly one thousand in 2016 ( 2.7 per 100,000 population) (Government of Canada 2017). However, the

\footnotetext{
${ }^{1}$ Department of Epidemiology, Biostatistics and Occupational Health, McGill University, Montréal, Canada.

${ }^{2}$ School of Epidemiology and Public Health, University of Ottawa, Ottawa, Canada.

${ }^{3}$ Department of Biology, University of Ottawa, Ottawa, Canada.

${ }^{4}$ Public Health Risk Sciences Division, National Microbiology Laboratory, Public Health Agency of Canada, Saint-Hyacinthe, Canada.

${ }^{5}$ Faculty of Veterinary Medicine, University of Montréal, Saint-Hyacinthe, Canada.
}

(c) Jean-Paul R. Soucy et al. 2018; Published by Mary Ann Liebert, Inc. This Open Access article is distributed under the terms of the Creative Commons Attribution Noncommercial License (http://creativecommons.org/licenses/by-nc/4.0/) which permits any noncommercial use, distribution, and reproduction in any medium, provided the original author(s) and the source are cited. 
true burden of LD in Canada is likely underestimated due to under-reporting in emerging areas (Ogden et al. 2015b).

Ecological niche modeling refers to a collection of statistical techniques based on correlating known presences of an organism with environmental variables to predict habitat suitability in a particular area. The proliferation of powerful, easy-to-use software packages such as MaxEnt and Genetic Algorithm for Rule Set Production (GARP) has led to the adoption of these models to predict risk areas for vectorborne diseases such as LD and malaria (Kulkarni et al. 2010, Mak et al. 2010). To date, many of the published models for the distribution of the black-legged tick and LD have focused on climatic variables, particularly temperature, producing relatively coarse maps with a resolution of kilometers (Estrada-Peña 1998, Brownstein et al. 2003, Khatchikian et al. 2012, Simon et al. 2014) or following larger administrative boundaries like census subdivisions (e.g., mean area $\left.=285 \mathrm{~km}^{2}\right)($ Ogden et al. 2008, Leighton et al. 2012) and counties (Ashley and Meentemeyer 2004). These models have formed the basis of successful predictions at a variety of spatial scales, including the presence of ticks in state parks (Brownstein et al. 2003), the density of nymphs collected from woodland rodents (Ogden et al. 2008), and abundance of questing ticks in small, forested sampling sites (Khatchikian et al. 2012).

Tick observations are collected through two types of surveillance: passive and active. In passive surveillance, ticks found by members of the public (usually attached to themselves or a pet) are submitted to public health officials, either directly or through physicians or veterinarians. Active surveillance occurs when researchers or public health authorities directly sample field sites by dragging (pulling a piece of cloth through potential tick habitat) or by trapping hosts such rodents, birds, and deer and examining them for ticks (Ogden et al. 2010). Active surveillance data have the advantage of being more geographically precise and more informative (e.g., recording tick density), but collecting these data is expensive and labor intensive, which often limits geographic coverage. Conversely, passive surveillance data are abundant and are routinely collected by public health authorities for tick identification and molecular detection of tick-borne pathogens. However, there is often uncertainty about the location of collection (due, in part, to submitter recall bias), a spatial bias toward collection near populated areas, and variation in regional collection programs (Johnson et al. 2004, Ogden et al. 2015a).

Broad-scale climate-based maps have obvious utility to public health (Ogden et al. 2008, Leighton et al. 2012), but they are unable to provide insight into the effects of localscale variation in key environmental variables such as the presence of woodland habitats that allow over-winter survival of ticks and provide habitat for tick hosts. Temperature, commonly used to predict the distribution of black-legged ticks at continental scales, varies little at the health unit level, the scale at which localized public health decisions are made. By comparison, land cover varies at the scale of meters, and data are available at much higher resolutions (e.g., $15 \mathrm{~m}$ ). Thus, in regions identified as broadly climatically suitable, high-resolution models built using land cover data are likely to offer more locally relevant predictions of vector occurrence. Passive surveillance data have been used to assess the broad-scale distribution of medically important ticks and associated diseases in the province of Ontario (Nelder et al. 2014) and to predict the locations of establishing black-legged tick populations in the province of Québec (Koffi et al. 2012), but to date the construction of risk models to guide public health action at a fine scale using passive surveillance data has not been attempted.

In this study, we developed a model for the occurrence of $I$. scapularis at high spatial resolution in the region of Ottawa, Ontario, Canada based on passive tick surveillance data collected by Ottawa Public Health between 2013 and 2016. Given the broad climatic suitability of the area (McPherson et al. 2017), we built the ecological niche model using variables derived from land cover and elevation potentially important to tick survival and establishment. We then tested the ability of the model to predict tick occurrence at 17 active surveillance sites sampled during the summer of 2017 and generated a validated map for potential I. scapularis habitat suitability in the region.

\section{Methods}

\section{Study area}

The study area was the City of Ottawa Health Unit, which comprises the city of Ottawa and its surrounding rural communities. Ottawa, located in the eastern portion of southern Ontario, is within the northern expanding frontier of LD risk in central Canada (Ogden et al. 2015b, Public Health Ontario $2015 b$ ). This region is dominated by farmland and forests, with elevation ranging from approximately $35-165 \mathrm{~m}$ above sea level.

\section{Data sources}

We obtained passive surveillance records from Ottawa Public Health, which collects voluntarily submitted ticks from residents within the City of Ottawa Health Unit. Ticks received by Ottawa Public Health between 2013 and 2015 were submitted through the Public Health Ontario laboratory to the National Microbiology Laboratory (NML), Winnipeg for species confirmation and testing for B. burgdorferi. In 2016, ticks were submitted by Ottawa Public Health directly to the University of Ottawa, where identification and testing followed protocols identical to those used at NML. The data were compiled to form a database of submitted ticks containing information on tick instar, stage of engorgement, result of testing for B. burgdorferi, and probable location of acquisition. Out of 540 submissions collected between April 2013 and December 2016, 306 were positively identified as I. scapularis. We retained georeferenced records with a high level of spatial certainty (e.g., specific address, park, or trail) on location of acquisition and excluded records with imprecise spatial locations (e.g., city level), implausible locations (e.g., highly urban), and locations outside of the study area. In total, 63 nonduplicate presence points were retained (Fig. 1).

Land cover and microclimatic variables potentially influencing the development, survival, and reproduction of I. scapularis at a fine scale were identified from published literature (Supplementary Table S1; Supplementary Data are available online at www.liebertpub.com/vbz). We focused on these variables as opposed to other possible variables (e.g., 
FIG. 1. Presence points $(n=63)$ used to train the Ixodes scapularis model in Ottawa, Canada. Rivers and lakes are shown in blue; population centers are outlined in black; the city of Ottawa and the suburban center of Kanata are labelled.

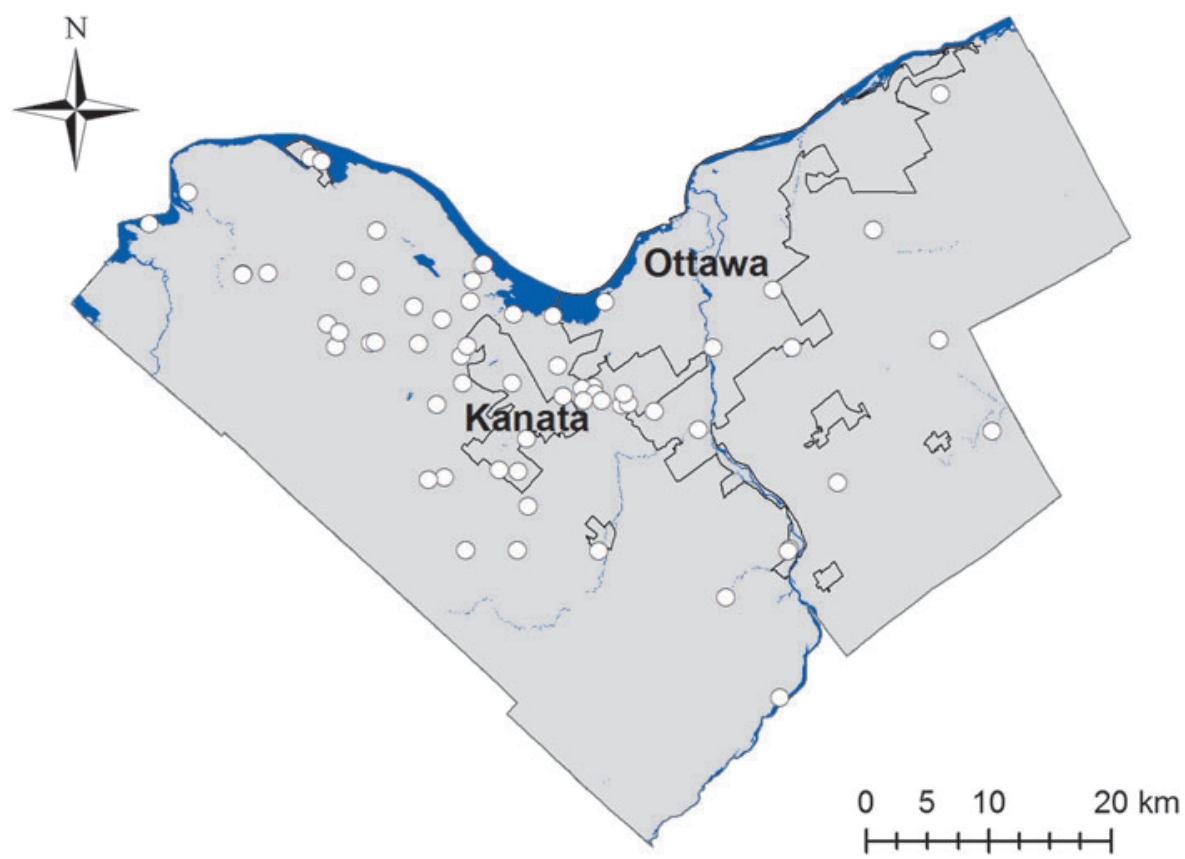

monthly vegetation indices) because they are available at very high resolution and are expected to remain relatively constant over the period of collection. To generate land cover variables, we used the Southern Ontario Land Resource Information System dataset, which is based on high-resolution (15 m) Landsat-7 satellite imagery classified into 30 categories (SOLRIS Technical Team 2015). We reclassified the 21 land cover types present in Ottawa into 13 categories as follows: 4 treed types (deciduous, mixed, coniferous, hedgerow), wetland, grassland/shrubland, tilled agricultural, undifferentiated rural, barren/other, built-up impervious, built-up pervious, roads/highways, and water (Supplementary Table S2). Land cover data were processed in ArcMap 10.4.1 (ESRI 2016) to calculate the proportion of each relevant habitat type within a $1000 \mathrm{~m}$ circular buffer, as well as the distance to these features (Supplementary Table S1). Elevation data were extracted from the Canadian Digital Elevation Model at $90 \mathrm{~m}$ resolution and resampled to the resolution of the land cover data $(15 \times 15 \mathrm{~m})$ (Natural Resources Canada 2017).

\section{Niche model development}

We applied a presence-only maximum entropy algorithm using MaxEnt version 3.4.1 (Phillips et al. 2006, 2017) [for a summary of the approach, see Elith et al. (2011)] to create the ecological niche model at a resolution of $15 \times 15 \mathrm{~m}$. The outcome of the model is a predicted habitat suitability value ranging from 0 to 1 . The model was trained using the passive surveillance dataset.

By default, MaxEnt contrasts the environmental characteristics of presence points with those of background points selected at random from the sampling region, under the assumption that presence points represent an unbiased sample of the region; however, the collection of presence points is frequently biased toward easily accessible areas (Phillips et al. 2009). Unless background points are sampled with the same bias as presence points, models trained on these data may exhibit spurious correlations induced by sampling bias
(Kramer-Schadt et al. 2013). In our study, all but one of the 63 presence points were found within $1 \mathrm{~km}$ of a road (although none were located directly on a road), with the frequency of observations decreasing exponentially with increasing distance from the road. Accordingly, background points were sampled from cells within $15-1000 \mathrm{~m}$ of a road with probability inversely proportional to distance from the road (Supplementary Methods; see also Supplementary Table S3 and Supplementary Figs. S1 and S2).

In our model, we considered feature class selection, regularization multiplier, and variable selection. These settings control the types of curves MaxEnt can fit (e.g., linear and quadratic), how tightly the model is fit to the data, and the choice of variables in the final model, respectively (for a detailed discussion of settings in MaxEnt, see Merow et al. 2013, Morales et al. 2017). Beginning with the full variable set, we used AICc (Warren and Seifert 2011, Wright et al. 2015) to determine the best combination of feature classes and regularization multiplier, as implemented in the enmSdm package (Smith 2017) in R 3.4.2 (R Core Team 2017). To improve the interpretability of the model, the variable set was reduced using regularized training gain as described by Yost et al. (2008) (Supplementary Methods). The final model was built using the full passive surveillance dataset.

We used three metrics to evaluate the importance of variables in the final model as follows: (1) permutation importance, obtained by randomly permuting the values of each variable in turn and measuring the decrease training AUC, (2) a jackknife procedure reporting the regularized training gain of models built using each variable individually, and (3) a jackknife procedure reporting the regularized training gain when each variable is excluded from the model in turn (Phillips 2017).

\section{Model evaluation}

Seventeen active surveillance sites (Fig. 2) were selected for drag sampling in 2017. Due to an unusually wet spring, sampling began in June and concluded in mid-August. Sites 


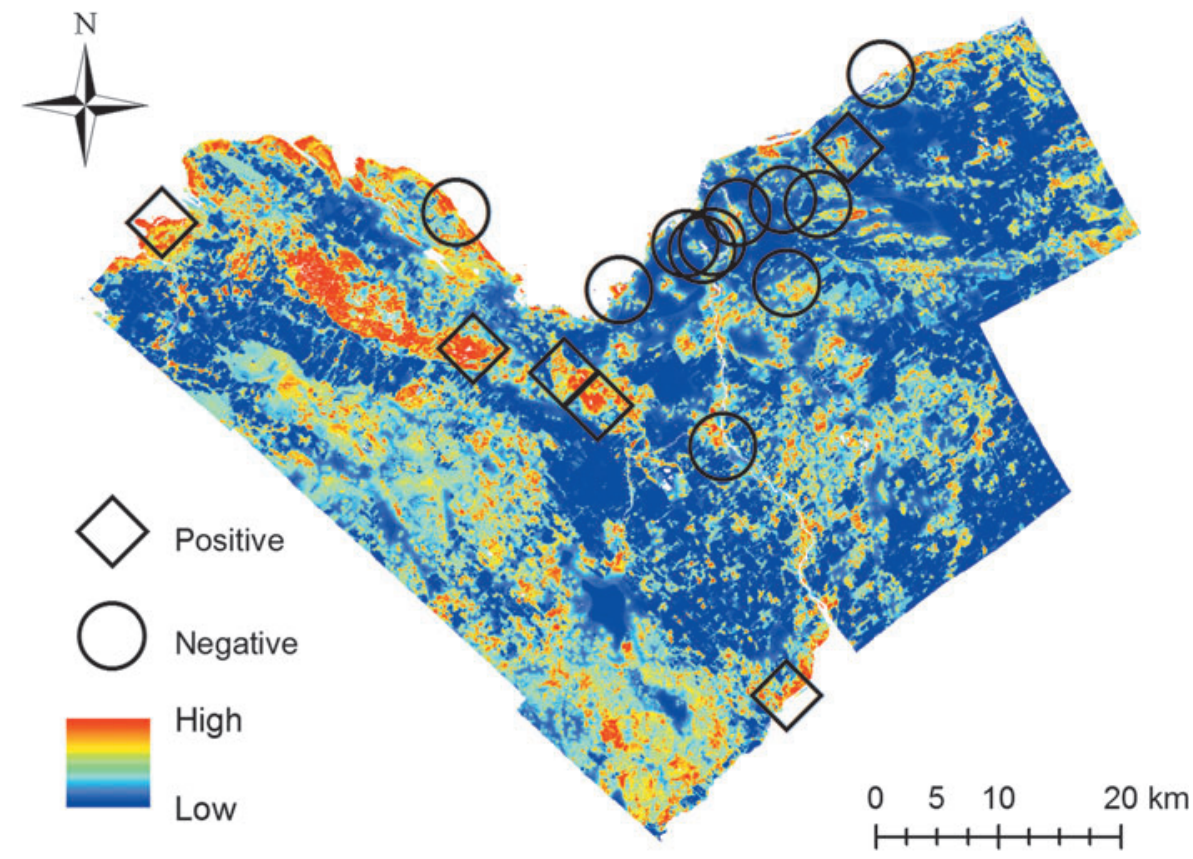

FIG. 2. Predicted habitat suitability for $I$. scapularis in Ottawa, Canada with tick presence or absence at active surveillance sites $(n=17)$. Positive sites $(n=6)$ are denoted with diamonds, and negative $(n=11)$ sites are denoted with circles. were chosen to reflect a range of environments across the study area, including both forested and urban locations. Sites included conservation areas, municipal parks, walking trails, and other green spaces. Each site was drag sampled for three person-hours with collectors checking drag sheets to collect and record ticks and note GPS coordinates every $50 \mathrm{~m}$, following the Public Health Ontario protocol (Public Health Ontario 2015a). In total, 810 georeferenced points corresponding to tick drags were recorded, of which $26(3.20 \%)$ were positive for at least one I. scapularis tick.

We evaluated our model using this independent active surveillance dataset. We used the area under the receiver operating characteristic curve (AUC), which ranges from 0 to 1 and may be interpreted as the probability that a randomly sampled presence point will have a higher predicted suitability than a randomly sampled absence point. In addition, we converted continuous predicted suitability values into binary predicted absence (0) and predicted presence (1) using a threshold that maximized the sum of sensitivity and specificity and reported sensitivity, specificity, positive predictive value, negative predictive value, and classification accuracy.

Evaluation was conducted by first classifying each of the 17 active surveillance sites as positive for tick presence $(n=6)$ if they had at least one tick observation and negative ( $n=11)$ otherwise (Fig. 2). Then, we used subsampling to reduce the impact of autocorrelation within sites in the evaluation dataset (Segurado et al. 2006, Kramer-Schadt et al. 2013). We randomly selected one presence point from each positive site and one absence point from each negative site, leaving 17 points in the evaluation dataset. This process was repeated 15 times, reporting the mean and standard error of the evaluation metrics. Model evaluation was performed using the dismo (Hijmans et al. 2017) package in R, which provides tools to calculate binary classification statistics such as sensitivity and AUC given a MaxEnt model and presence/absence data.

\section{Results}

\section{Niche model development}

The best fit model based on AICc employed only hinge features with a regularization multiplier of 1.5. After variable selection, the final model contained the following variables: distance from deciduous forest (decreasing suitability with increasing distance), distance from coniferous forest (decreasing with increasing distance), distance from treed areas (including hedgerows) (decreasing with increasing distance), distance from tilled agricultural land (low suitability in/adjacent to agricultural land), proportion of tilled agricultural/undifferentiated rural land (suitability peaks at low-to-moderate proportion), proportion of water (increasing with increasing proportion), and elevation (peaks at moderate elevation) (Supplementary Fig. S3). Distance from treed areas and distance from tilled agricultural land were the most important variables according to permutation importance (Table 1).

\section{Model evaluation}

Our niche model of tick occurrence developed using passive surveillance data was evaluated using subsampling on an active surveillance dataset. The model demonstrated good discrimination of positive and negative sites for I. scapularis presence (AUC $=0.878 \pm 0.019$, classification accuracy $=0.835 \pm 0.020)$ and greater sensitivity $(0.956 \pm 0.026)$ than specificity $(0.769 \pm 0.028)$. The negative predictive value of the model $(0.972 \pm 0.015)$ exceeded the positive predictive value $(0.705 \pm 0.026)$.

\section{Final model}

Our final model predicts the suburban and rural areas in the western and southwestern areas of the City of Ottawa Health Unit to be most highly suitable for tick occurrence (Fig. 2). This coincides with heavily forested regions in Ottawa's west end 
Table 1. Three Measures of Model Contribution for Covariates in the Ixodes scapularis Model

Full model regularized training gain: 0.7371

\begin{tabular}{lccc}
\hline Variable & $\begin{array}{c}\text { Permutation } \\
\text { importance }\end{array}$ & $\begin{array}{c}\text { Gain without } \\
\text { variable }\end{array}$ & $\begin{array}{c}\text { Gain with } \\
\text { only variable }\end{array}$ \\
\hline Distance from treed (including hedgerows) & 36.8 & 0.650 & 0.433 \\
Distance from tilled agricultural & 35.9 & 0.672 & 0.211 \\
Proportion of water & 10 & 0.656 & 0.144 \\
Proportion of tilled agricultural/undifferentiated rural & 5.6 & 0.698 & 0.112 \\
Distance from coniferous forest & 5.3 & 0.704 & 0.128 \\
Distance from deciduous forest & 3.8 & 0.724 & 0.305 \\
Elevation & 2.6 & 0.717 & 0.039 \\
\hline
\end{tabular}

and southwest localities compared to the more agricultural eastern areas. However, some localized areas of high suitability were also predicted across the study area, especially along the Rideau River. These areas offer a potential corridor to facilitate the dispersal of ticks northward from highly infested regions southwest of the Ottawa Public Health Unit, which serve as the focal point of LD in Ontario (Public Health Ontario 2015b).

\section{Discussion}

In this study, we developed a high-resolution ecological niche model for the occurrence of I. scapularis in the region of Ottawa, which is on the northern frontier of LD in central Canada (Ogden et al. 2015b, Public Health Ontario 2015b). The model was built using passive tick surveillance data collected by the local public health authority and a variety of land cover and microclimatic variables, including distance from forests and elevation. The use of passive surveillance data to build models of relevance to public health decision-making at fine scales is not well established, as these data are likely to reflect sampling bias (Beck et al. 2014). Thus, we evaluated the model using an independent, active surveillance dataset collected at 17 sites relevant to human tick exposure in Ottawa, Ontario.

The model effectively distinguished sites that were positive for tick presence from sites that were negative. Although sensitivity exceeded specificity, negative predictive value exceeded positive predictive value due to the low overall prevalence of ticks at sampling sites (6/17). Our choice of threshold (maximizing the sum of sensitivity and specificity) may have contributed to this apparent bias toward overprediction; however, we believe that overprediction is preferable from a public health standpoint to underprediction in an area of emerging risk. In addition, we would expect the appearance of overprediction, as active surveillance may not detect emerging tick populations in which tick density is very low (Ogden et al. 2014a). This is an observation common to other areas of I. scapularis range expansion in Canada (Gabriele-Rivet et al. 2017). The month of sampling may also affect detection, as the abundance of each life stage varies seasonally. The choice of sampling period, June to August, coincides with a peak in the activity of the nymphal stage (the stage most associated with human risk) (Falco et al. 1999) and the period when most cases of LD are reported in Ontario (Ogden et al. 2015b). Furthermore, in zones of emergence of I. scapularis populations, it would be expected that not all locations that are environmentally suitable for the ticks have, to date, become occupied by tick populations. Overall, our findings show that ecological niche models based on passive surveillance data produce accurate fine-scale predictions of I. scapularis presence/absence at the local level.

Further validation of our model was given by the observed relationships between fine-scale landscape features and predicted tick suitability, which are largely consistent with published literature. Although evidence on the importance of forests at the municipal scale is mixed (Brownstein et al. 2005, Leighton et al. 2012), our study confirms the central importance of forests to tick establishment at fine scales, with both deciduous forests (considered the classical habitat of the black-legged tick) and coniferous forests appearing in the model (Killilea et al. 2008, Stone et al. 2017). Although agricultural fields are inhospitable to ticks due to the risk of desiccation from low canopy (Bertrand and Wilson 1996, Das et al. 2002), low-to-moderate intensity agriculture creates fragmented forest patches promoting the abundance of ticks and their hosts (Nupp and Swihart 1996, Allan et al. 2003, Brownstein et al. 2005), which is consistent with our model showing higher tick suitability in areas with low-tomoderate agricultural intensity. Finally, our model demonstrated a positive relationship between predicted suitability and surrounding proportion of water, possibly due to the habitat requirements of key tick hosts such as white-tailed deer (Bunnell et al. 2003, Chen et al. 2015).

The accessibility of remote sensing data has resulted in many national and continental scale predictive models of tick and mosquito vector distribution for diseases of public health importance (Porretta et al. 2013, Ogden et al. 2014b), with several national-scale risk maps for I. scapularis being available (Ogden et al. 2008, Leighton et al. 2012). Highresolution models such as the one presented in this study are likely to offer even more relevant guidance for local public health decision-making (Kulkarni et al. 2010). The main application of this model is to assist in the identification of current risk areas for $\mathrm{LD}$ and areas of potential tick emergence to prioritize for active surveillance and public health messaging. More generally, this model contributes to the literature on the determinants of tick and LD emergence at a local scale. However, inference of LD risk at this scale is impeded by the fact that risk is a complex function of entomological risk, human land use, and the proportion of B. burgdorferi infected ticks, all of which are affected by different factors (Rand et al. 1996, Brownstein et al. 2005, Werden et al. 2014). Habitat suitability, which is the component predicted by our model, is just one piece of the puzzle. Nevertheless, to date in this region of Canada, the arrival of I. scapularis populations has 
been followed by the arrival of B. burgdorferi transmission cycles and progressive increases in tick abundance and B. burgdorferi infection prevalence (Ogden et al. 2013).

Finally, risk maps such as the one produced in the current study should be applied and interpreted cautiously, since actual tick presence may differ from predicted values for a variety of reasons unaccounted for in the model, such as barriers to host dispersal, human habitat modification, and seasonal variation (Estrada-Peña et al. 2013). In addition, there is a low baseline risk of tick encounter anywhere that ticks may be dispersed by their hosts, such as birds and deer (Madhav et al. 2004, Ogden et al. 2008). Dispersal into environmentally unsuitable habitats presents major challenge in predicting the localized distribution of a generalist parasite such as $I$. scapularis by introducing noise into the modeling process. Given that there is no way to account for local dispersal events simply by measuring environmental characteristics, it is even more significant that our model is nonetheless able to distinguish positive and negative sites for tick presence.

\section{Conclusions}

Despite the limitations of passive surveillance data, our study demonstrated the utility of these data to model localscale environmental risk for a tick vector of human disease and was validated by active surveillance at sites of public health interest. The types of environmental data used in this study are widely available, allowing for the possibility of applying this methodology to disease vectors in other locales. Our study highlights the benefit of reinforcing research collaborations between public health authorities and researchers, as passive surveillance data and ecological niche models based on them have a key role to play in the detection and mitigation of emerging diseases.

Our findings also raise concerns about the future of LD risk in the municipality of Ottawa. Since warming temperatures have rendered the area broadly suitable from a climatic perspective (McPherson et al. 2017) and suitable habitat exists surrounding populated areas, the abundance of ticks and incidence of LD are likely to increase in the future. Therefore, efforts must be made to increase awareness of the disease and basic tick-bite prevention measures among healthcare providers and members of the public, as LD risk expands in Ontario and the rest of Canada.

\section{Acknowledgments}

The authors thank Ottawa Public Health for providing the passive tick surveillance data used in this study and Charles Thickstun, Merlin Caron-Levesque, and Itai Malkin for their contributions to field work. This work was partially supported by a grant to MAK from the Canadian Institutes for Health Research. JTK is grateful for Discovery Grant support from the Natural Sciences and Engineering Research Council of Canada and to the University of Ottawa for funding through the University Research Chair in Macroecology and Conservation.

\section{Author Disclosure Statement}

The authors state that no competing financial interests exist.

\section{References}

Allan BF, Keesing F, Ostfeld RS. Effect of forest fragmentation on lyme disease risk. Conserv Biol 2003; 17: 267-272.

Ashley ST, Meentemeyer V. Climatic analysis of lyme disease in the United States. Clim Res 2004; 27:177-187.

Beck J, Böller M, Erhardt A, Schwanghart W. Spatial bias in the GBIF database and its effect on modeling species' geographic distributions. Ecol Inform 2014; 19:10-15.

Bertrand MR, Wilson ML. Microclimate-dependent survival of unfed adult Ixodes scapularis (Acari: Ixodidae) in nature: Life cycle and study design implications. J Med Entomol 1996; 33: 619-627.

Brownstein JS, Holford TR, Fish D. A climate-based model predicts the spatial distribution of the lyme disease vector Ixodes scapularis in the United States. Environ Health Perspect 2003; 111:1152-1157.

Brownstein JS, Skelly DK, Holford TR, Fish D. Forest fragmentation predicts local scale heterogeneity of lyme disease risk. Oecologia 2005; 146:469-475.

Bunnell JE, Price SD, Das A, Shields TM, et al. Geographic information systems and spatial analysis of adult Ixodes scapularis (Acari: Ixodidae) in the Middle Atlantic Region of the U.S.A. J Med Entomol 2003; 40:570-576.

Chen D, Wong H, Belanger P, Moore K, et al. Analyzing the correlation between deer habitat and the component of the risk for lyme disease in Eastern Ontario, Canada: A GISbased approach. ISPRS Int J Geo-Information 2015; 4:105123.

Das A, Lele SR, Glass GE, Shields T, et al. Modelling a discrete spatial response using generalized linear mixed models: Application to lyme disease vectors. Int J Geogr Inf Sci 2002; 16:151-166.

Elith J, Phillips SJ, Hastie T, Dudík M, et al. A statistical explanation of MaxEnt for ecologists. Divers Distrib 2011; 17: 43-57.

ESRI. ArcGIS Desktop: Release 10.4.1. 2016. Available at www.esri.com

Estrada-Peña A. Geostatistics and remote sensing as predictive tools of tick distribution: A cokriging system to estimate $I x$ odes scapularis (Acari: Ixodidae) habitat suitability in the United States and Canada from advanced very high resolution radiometer satellite imagery. J Med Entomol 1998; 35:989995.

Estrada-Peña A, Gray JS, Kahl O, Lane RS, et al. Research on the ecology of ticks and tick-borne pathogens-methodological principles and caveats. Front Cell Infect Microbiol 2013; 3:29.

Falco RC, McKenna DF, Daniels TJ, Nadelman RB, et al. Temporal relation between Ixodes scapularis abundance and risk for lyme disease associated with erythema migrans. Am J Epidemiol 1999; 149:771-776.

Gabriele-Rivet V, Koffi JK, Pelcat Y, Arsenault J, et al. A risk model for the lyme disease vector Ixodes scapularis (Acari: Ixodidae) in the Prairie provinces of Canada. J Med Entomol 2017; 54:862-868.

Government of Canada. Surveillance of Lyme disease. 2017. Available at http://healthycanadians.gc.ca/diseases-conditionsmaladies-affections/disease-maladie/lyme/surveillance-eng.php

Hijmans RJ, Phillips S, Leathwick J, Elith J. Dismo: Species Distribution Modeling: R package version 1.1-4. 2017. Available at https://cran.r-project.org/package=dismo

Johnson JL, Ginsberg HS, Zhioua E, Whitworth UG, et al. Passive tick surveillance, dog seropositivity, and incidence of 
human lyme disease. Vector Borne Zoonotic Dis 2004; 4: 137-142.

Khatchikian CE, Prusinski M, Stone M, Backenson PB, et al. Geographical and environmental factors driving the increase in the lyme disease vector Ixodes scapularis. Ecosphere 2012; 3:art85.

Killilea ME, Swei A, Lane RS, Briggs CJ, et al. Spatial dynamics of lyme disease: A review. Ecohealth 2008; 5:167195.

Koffi JK, Leighton PA, Pelcat Y, Trudel L, et al. Passive surveillance for I. scapularis ticks: Enhanced analysis for early detection of emerging lyme disease risk. J Med Entomol 2012; 49:400-409.

Kramer-Schadt S, Niedballa J, Pilgrim JD, Schröder B, et al. The importance of correcting for sampling bias in MaxEnt species distribution models. Divers Distrib 2013; 19:13661379.

Kulkarni MA, Desrochers RE, Kerr JT. High resolution niche models of malaria vectors in Northern Tanzania: A new capacity to predict malaria risk? PLoS One 2010; 5: e9396.

Leighton PA, Koffi JK, Pelcat Y, Lindsay LR, et al. Predicting the speed of tick invasion: An empirical model of range expansion for the lyme disease vector Ixodes scapularis in Canada. J Appl Ecol 2012; 49:457-464.

Madhav NK, Brownstein JS, Tsao JI, Fish D. A dispersal model for the range expansion of blacklegged tick (Acari: Ixodidae). J Med Entomol 2004; 41:842-852.

Mak S, Morshed M, Henry B. Ecological niche modeling of lyme disease in British Columbia, Canada. J Med Entomol 2010; 47:99-105.

McPherson M, García-García A, Cuesta-Valero FJ, Beltrami H, et al. Expansion of the lyme disease vector Ixodes scapularis in Canada inferred from CMIP5 climate projections. Environ Health Perspect 2017; 125:1-9.

Merow C, Smith MJ, Silander JA. A practical guide to MaxEnt for modeling species' distributions: What It does, and why inputs and settings matter. Ecography (Cop) 2013; 36:10581069.

Morales NS, Fernández IC, Baca-González V. MaxEnt's parameter configuration and small samples: Are we paying attention to recommendations? A systematic review. PeerJ 2017; 5:e3093.

Natural Resources Canada. Canadian Digital Elevation Model: Product Specifications - Edition 1.1; Ottawa, ON: Natural Resources Canada, 2017.

Nelder MP, Russell C, Lindsay LR, Dhar B, et al. Populationbased passive tick surveillance and detection of expanding foci of blacklegged ticks Ixodes scapularis and the Lyme Disease Agent Borrelia burgdorferi in Ontario, Canada. PLoS One 2014; 9:e105358.

Nupp TE, Swihart RK. Effect of Forest Patch Area on Population Attributes of White-Footed Mice (Peromyscus leucopus) in Fragmented Landscapes. Can J Zool 1996; 74 : 467-472.

Ogden NH, St-Onge L, Barker IK, Brazeau S, et al. Risk maps for range expansion of the lyme disease vector, Ixodes scapularis, in Canada now and with climate change. Int $\mathrm{J}$ Health Geogr 2008; 7:24.

Ogden NH, Bouchard C, Kurtenbach K, Margos G, et al. Active and passive surveillance and phylogenetic analysis of Borrelia burgdorferi elucidate the process of lyme disease risk emergence in Canada. Environ Health Perspect 2010; 118: 909-914.
Ogden NH, Lindsay LR, Leighton PA. Predicting the Rate of Invasion of the Agent of lyme disease Borrelia burgdorferi. J Appl Ecol 2013; 50:510-518.

Ogden NH, Koffi JK, Pelcat Y, Lindsay LR. Environmental risk from lyme disease in central and Eastern Canada: A summary of recent surveillance information. Canada Commun Dis Rep 2014a; 40:74-82.

Ogden NH, Milka R, Caminade C, Gachon P. Recent and projected future climatic suitability of North America for the Asian Tiger Mosquito Aedes albopictus. Parasit Vectors 2014b; 7:532.

Ogden NH, Feil EJ, Leighton PA, Lindsay LR, et al. Evolutionary aspects of emerging lyme disease in Canada. Appl Environ Microbiol 2015a; 81:7350-7359.

Ogden NH, Koffi JK, Lindsay LR, Fleming S, et al. Surveillance for Lyme Disease in Canada, 2009 to 2012. Canada Commun Dis Rep 2015b; 41:132-145.

Phillips SJ. A Brief Tutorial on Maxent. 2017. Available at https:// biodiversityinformatics.amnh.org/open_source/maxent/Maxent_ tutorial2017.pdf

Phillips SJ, Anderson RP, Schapire RE. Maximum entropy modeling of species geographic distributions. Ecol Modell 2006; 190:231-259.

Phillips SJ, Dudík M, Elith J, Graham CH, et al. Sample selection bias and presence-only distribution models: Implications for background and pseudo-absence data. Ecol Appl 2009; 19:181-197.

Phillips SJ, Anderson RP, Dudík M, Schapire RE, et al. Opening the black box: An open-source release of maxent. Ecography (Cop) 2017; 40:887-893.

Porretta D, Mastrantonio V, Amendolia S, Gaiarsa S, et al. Effects of global changes on the climatic niche of the tick Ixodes ricinus inferred by species distribution modelling. Parasit Vectors 2013; 6:271.

Public Health Ontario. Tick Dragging: Standard Operating Procedure. Toronto, ON: Queen's Printer for Ontario, 2015a.

Public Health Ontario. Vector-Borne Diseases 2014 Summary Report. Toronto, ON: Queen's Printer for Ontario, $2015 b$.

R Core Team. R: A Language and Environment for Statistical Computing. Vienna, Austria: R Foundation for Statistical Computing, 2017.

Rand PW, Lacombe EH, Smith, Jr. RP, Gensheimer K, et al. Low seroprevalence of human lyme disease near a focus of high entomologic risk. Am J Trop Med Hyg 1996; 55:160164.

Schauber EM, Ostfeld RS, Evans, Jr. AS. What is the best predictor of annual lyme disease incidence: Weather, mice, or acorns? Ecol Appl 2005; 15:575-586.

Segurado P, Araujo MB, Kunin WE. Consequences of spatial autocorrelation for niche-based models. J Appl Ecol 2006; 43:433-444.

Simon JA, Marrotte RR, Desrosiers N, Fiset J, et al. Climate change and habitat fragmentation drive the occurrence of Borrelia burgdorferi, the agent of lyme disease, at the northeastern limit of its distribution. Evol Appl 2014; 7:750764.

Smith AB. enmSdm: Tools for modeling species niches and distributions: R package version 0.1.0.0. 2017. Available at www.earthskysea.org

SOLRIS Technical Team. Southern Ontario Land Resource Information System 2.0: Data Specifications. Peterborough, ON: Ministry of Natural Resources and Forestry, 2015. 
Stone BL, Tourand Y, Brissette CA. Brave New Worlds: The Expanding Universe of Lyme Disease. Vector Borne Zoonotic Dis 2017; 17:619-629.

Warren DL, Seifert SN. Ecological Niche Modeling in Maxent: The Importance of Model Complexity and the Performance of Model Selection Criteria. Ecol Appl 2011; 21: 335-342.

Werden L, Barker IK, Bowman J, Gonzales EK, et al. Geography, deer, and host biodiversity shape the pattern of lyme disease emergence in the thousand islands Archipelago of Ontario, Canada. PLoS One 2014; 9:e85640.

Wright AN, Hijmans RJ, Schwartz MW, Shaffer HB. Multiple sources of uncertainty affect metrics for ranking conservation risk under climate change. Divers Distrib 2015; 21:111-122.
Yost AC, Petersen SL, Gregg M, Miller R. Predictive modeling and mapping sage grouse (Centrocercus urophasianus) nesting habitat using maximum entropy and a long-term dataset from Southern Oregon. Ecol Inform 2008; 3:375-386.

Address correspondence to: Manisha A. Kulkarni School of Epidemiology and Public Health University of Ottawa 600 Peter Morand Cresent, Room 217B

Ottawa, ON, K1G5Z3

Canada

E-mail: manisha.kulkarni@uottawa.ca 